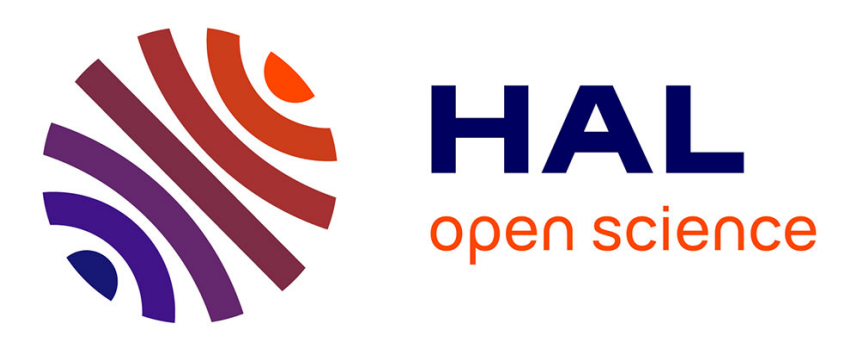

\title{
Enquêtes épidémiologiques en milieu de travail: quelques bases pour le médecin du travail
}

Alexis Descatha, Béatrice Geoffroy-Perez, Annette Leclerc, Marcel Goldberg

\section{To cite this version:}

Alexis Descatha, Béatrice Geoffroy-Perez, Annette Leclerc, Marcel Goldberg. Enquêtes épidémiologiques en milieu de travail: quelques bases pour le médecin du travail. Archives des Maladies Professionnelles et de L'Environnement, 2005, 66 (4), pp.343-351. inserm-00141283

\section{HAL Id: inserm-00141283 https://www.hal.inserm.fr/inserm-00141283}

Submitted on 12 Apr 2007

HAL is a multi-disciplinary open access archive for the deposit and dissemination of scientific research documents, whether they are published or not. The documents may come from teaching and research institutions in France or abroad, or from public or private research centers.
L'archive ouverte pluridisciplinaire HAL, est destinée au dépôt et à la diffusion de documents scientifiques de niveau recherche, publiés ou non, émanant des établissements d'enseignement et de recherche français ou étrangers, des laboratoires publics ou privés. 
Enquêtes épidémiologiques en milieu de travail: quelques bases pour le médecin du travail

A. Descatha $\left({ }^{1,2}\right)$, B. Geoffroy-Perez $\left({ }^{3}\right)$, A. Leclerc $\left({ }^{1}\right)$, M. Goldberg $\left({ }^{1,3}\right)$

( ${ }^{1}$ ) INSERM U687-IFR 69, Hôpital National de Saint-Maurice, 14 rue du val d'Osnes, 94415 Saint-Maurice CEDEX.

$\left(^{2}\right)$ Unité de pathologie professionnelle, de santé de travail, et d'insertion. Hôpital R. Poincaré, AP-HP, 104 bd R. Poincaré, 92380 Garches.

$\left({ }^{3}\right)$ Département Santé Travail, InVS, 12 rue du Val d'Osnes, 94415 Saint-Maurice CEDEX.

Correspondance et adresse : Alexis Descatha, à l'adresse ci-dessus.

Titre courant: Bases pratiques des études épidémiologiques

Mots clés: épidémiologie. formation 


\section{Résumé}

L'épidémiologie a pris une place prépondérante dans la recherche en milieu de travail. Le médecin du travail n'a pas toujours toutes les clés pour comprendre les bases des études épidémiologiques, d'y participer, voire de les organiser.

L'objectif de ce travail est d'en rappeler les principes en donnant les étapes de réalisation d'une étude, de la formulation de la question à la publication, en insistant sur la nécessité de travailler en équipe avec des spécialistes de l'épidémiologie des risques professionnels. Nous allons nous pencher particulièrement sur la réalisation du protocole d'une étude épidémiologique, qui en planifie toutes les étapes essentielles.

Après une étape bibliographique indispensable, la mise en place d'un protocole complet va permettre de rappeler le contexte de l'étude épidémiologique, d'en définir l'objectif principal et les moyens mis en œuvre pour l'atteindre. Le protocole définit le type d'enquête, la population étudiée et les données à analyser. Le protocole donne également les différents aspects logistiques de l'étude. Les bases de l'analyse et l'interprétation sont enfin rapidement abordées. Les différentes étapes sont illustrées par un exemple concret.

\section{Summary}

Epidemiology has taken an important part of research on occupational risk. The occupational health physician does not have all clues to understand, participate and organize epidemiologic studies on the workplace.

The purpose of this work is to remind and clarify major steps of an epidemiologic survey on the workplace, especially with collaborative work occupational epidemiological unit. Study protocol, which included all major steps of a survey, was detailed.

After complete literature review, the protocol of the study must include background, purpose of the study, and the methods to achieve it. The protocol also approached the data collection and other material questions. Results analysis and interpretation were mentioned. One example was also given. 
L'épidémiologie a pris une place prépondérante dans la recherche en milieu de travail. Les médecins du travail sont souvent sollicités pour recueillir des données sur les expositions et les pathologies professionnelles, pour évaluer les risques, voire pour organiser des projets de recherche. Les enquêtes épidémiologiques, associant le médecin du travail et l'épidémiologiste dans un projet multidisciplinaire, permettent d'améliorer les connaissances sur les risques professionnels. Les études sont souvent publiées dans les revues de médecine du travail de formation médicale continue. Or le médecin du travail n'a pas toujours toutes les clés pour comprendre et participer pleinement à ces études.

L'objectif de ce synopsis est de rappeler les bases des enquêtes épidémiologiques en milieu professionnel ou, tout du moins, de préciser les principes permettant la lecture critique des enquêtes publiées ; il s'adresse au médecin du travail participant, voire initiant, des travaux de recherche dans le contexte français. Il ne se veut pas exhaustif car il existe des ouvrages plus complets $(1,2)$. Nous illustrerons notre propos d'un exemple tiré d'une enquête sur les troubles musculo-squelettiques conduite par l'ANACT (Agence nationale pour l'amélioration des conditions de travail), l'INSERM Unité 88 (Institut national pour la santé et la recherche médicale) et d'autres partenaires réunis dans le Groupe de travail sur les gestes répétitifs (3).

\section{Préalable}

Avant de commencer une enquête, l'étude de la bibliographie permet de faire le point sur ce qui est connu et/ou évoqué, pour conclure à la pertinence de l'enquête et de connaître les difficultés rencontré par les auteurs pour les intégrer dans la construction de l'enquête. De nombreuses bases de données bibliographiques sont actuellement disponibles, dont certaines sont en ligne sur Internet (PUBMED), d'autres plus spécialisées sont accessibles uniquement dans les bibliothèques spécialisées (OVID, PASCAL, EMBASE...). La recherche se fait aussi sur des études non publiées (mémoire, thèse, rapport ...), en s'adressant à des équipes d'épidémiologie connaissant bien le domaine.

\section{Mise en place : Protocole}

La première étape d'une enquête consiste dans la rédaction du protocole d'étude. Il s'agit d'un document où sont consignés de façon clair le contexte, les objectifs et les moyens de l'étude. Le protocole permet de limiter au maximum les erreurs qui pourraient survenir, ainsi que de s'assurer que les moyens mis en œuvre permettront de répondre à l'objectif de l'étude.

Dans le domaine professionnel, il existe des recommandations françaises de bonnes pratiques (rédigées par l'Association des épidémiologistes de langue française ou ADELF avec une contribution de l'Association pour le développement des études et recherches épidémiologiques sur la santé au travail ou ADEREST), qui détaillent les modalités de rédaction d'un protocole (4). Le protocole comporte le titre de l'étude, le contexte humain administratif (investigateurs, financeurs, sous-traitants 
...), le contexte scientifique (littérature, études pilotes ou préliminaires) ainsi que les objectifs de l'enquête ; la méthodologie complète (type d'étude choisi, critères d'inclusion et de jugement), le détail du recueil de données (concernant la maladie, l'exposition et les autres variables, ainsi que leur caractère éventuellement nominatif), l'utilisation qui en sera faite, notamment la stratégie d'analyse envisagée, leur classement et leur protection ainsi que les modalités pratiques de réalisation de l'enquête (vérifications prévues, calendrier, budget et protection des personnes).

Le protocole reprend notamment le contexte, les hypothèses et la méthodologie utilisée pour éprouver ces dernières.

\section{Contexte}

Du point de vue de l'implication du médecin du travail, il existe deux situations typiques.

La première concerne la suspicion d'un risque professionnel potentiel. Des structures épidémiologiques spécialisées en risques professionnels, souvent mandatées par les autorités sanitaires, se chargent d'étudier ce risque. Elles invitent le médecin du travail à participer, pour la détermination pratique des expositions et des maladies, par l'intermédiaire de questionnaires, d'études de poste ou d'examens cliniques. Bien qu'en général le médecin du travail soit modérément impliqué dans l'ensemble des étapes de l'enquête, il est informé du contexte, du ou des objectif(s) ainsi que des méthodes employées dans le but d'une meilleure implication dans l'étude. Il doit être informé de la suite donnée à l'enquête, notamment des résultats et des conclusions de celle-ci.

Une autre situation correspond à celle où les médecins du travail identifient un dysfonctionnement dans une ou plusieurs entreprises dont ils ont la charge. Cela peut être une situation à risque marquée par un accident du travail ou bien un nombre observé de maladies professionnelles jugé anormalement élevé. La demande peut émaner du médecin du travail lui-même, d'autres partenaires de l'entreprise, ou d'un groupe de médecins du travail.

Dans les études publiées, le contexte de la réalisation de l'étude est présenté dans la première partie de l'introduction.

Quelle que soit la situation, les hypothèses de départ seront replacées dans leur contexte social et scientifique, avec une recherche bibliographique aussi exhaustive que possible sur le sujet.

Dans notre exemple, le contexte de mise en place d'une étude épidémiologique était celui de l'augmentation du nombre de cas de troubles musculo-squelettiques du membre supérieur reconnus en maladie professionnelle. Dans cette étude, les médecins du travail ont été sollicités par une unité d'épidémiologie spécialisée en risques professionnels pour l'administration de questionnaires et la réalisation d'un examen clinique standardisé (5). 


\section{Objectifs}

La détermination des objectifs est une étape clé dans l'élaboration d'une étude. L'objectif représente l'essence d'une enquête. Il détermine presque entièrement les modalités de l'étude. L'objectif doit répondre à la question soulevée précédemment. À cet objectif dit principal, peuvent s'adjoindre des objectifs secondaires, en nombre nécessairement restreint. Le recours à l'outil statistique ne peut en effet se concevoir qu'à travers un nombre limité d'hypothèses clairement définies à l'avance. Lors d'enquêtes multicentriques coordonnées par des équipes d'épidémiologie, il n'est pas rare que celles-ci englobent plusieurs questions autour d'un objectif précis, voire même implique le recueil de nombreuses données constituant ainsi une base pouvant être utilisée pour d'autres projets d'études ultérieures. Néanmoins les enquêtes coordonnées en milieu de travail doivent répondre généralement à une seule question posée.

La mise au point d'objectifs nécessite de prendre en compte trois axes :

- utilité et intérêt de l'étude ;

- faisabilité ;

- possibilité de conclure.

Ces trois axes doivent être analysés, permettant d'éviter des enquêtes inutiles.

La mise au point d'objectifs peut nécessiter la réalisation d'études préliminaires sur des effectifs réduits, appelées études pilotes ou de faisabilité.

Dans un article original, les objectifs sont présentés dans le dernier paragraphe de l'introduction.

L'étude que nous citons en exemple était issue d'un vaste recueil de données sur les TMS. Elle avait pour objectif d'identifier des facteurs professionnels associés à l'incidence du syndrome du nerf ulnaire au coude dans une population exposée aux gestes répétitifs $(3,5)$.

\section{Moyens}

La vérification de l'adéquation entre les moyens nécessaires et les moyens disponibles doit se faire au moment de la définition des objectifs.

Ces moyens sont de quatre sortes : humains, financiers, techniques et réglementaires.

\section{Moyens humains.}


Dans la première situation, le médecin du travail est sollicité par les unités d'épidémiologie. Il répond en fonction de ses priorités (facteur temps) et de celles du service de médecine du travail. Il peut demander à ne participer que si certaines conditions sont remplies: information sur le déroulement et les conditions de l'étude, mais aussi besoin éventuel de formation épidémiologique et/ou clinique dans le domaine couvert par l'enquête, pour lui-même et pour les autres personnes participant à l'enquête (infirmières du travail, secrétaires médicales ...)

Dans la deuxième situation, les moyens humains peuvent être un facteur limitant majeur: au-delà des aspects financiers dont nous reparlerons, le médecin du travail doit disposer du temps nécessaire aux investigations, voire à l'organisation et l'analyse des données de l'étude.

Des obstacles sociaux divers peuvent interférer avec la réalisation d'une enquête épidémiologique (désaccord d'un partenaire actif de l'entreprise, en particulier).

Dans ces deux types de situations, le travail est réalisé au sein d'une équipe pluridisciplinaire. Le médecin du travail peut travailler avec des services de recherche ou de surveillance spécialisés dans ce type d'étude ou des services de pathologies professionnelles hospitalo-universitaires.

\section{Moyens financiers.}

Une différence primordiale entre les deux types de situations que nous avons décrites réside dans les sources de financement et la différence de budget attribué à l'étude en projet. Les objectifs de santé publique nécessitent généralement des enquêtes épidémiologiques à grande échelle, donc des moyens financiers proportionnels aux effectifs impliqués et aux objectifs poursuivis. A contrario, les enquêtes organisées et coordonnées par le médecin de travail à l'échelle de l'entreprise portent en général sur des effectifs plus restreints et réclament a priori de moindres moyens financiers. Le médecin du travail peut par ailleurs répondre à des appels d'offre pour des enquêtes à grande échelle, avec généralement la nécessité de travailler avec des équipes d'épidémiologie (ce qui simplifie les demandes de financement). Enfin, dans le cas où une étude épidémiologique serait entreprise par un médecin du travail à plus petite échelle, le financement peut être interne à l'entreprise ou provenir d'un organisme extérieur avec lequel l'entreprise aura passé un accord, mais qui pourra être source de conflit d'intérêt.

\section{Moyens techniques et analyse statistique.}

Dans le protocole, l'analyse des résultats doit être prévue, notamment sur le plan pratique des moyens affectés à cet effet. Néanmoins, en dehors des enquêtes purement descriptives, le principe de l'analyse des résultats repose sur des notions simples : existe-t-il des différences entre ce qui est 
observé et ce qui est attendu et, le cas échéant, ceci est-il le fruit du hasard ou bien d'une différence réelle?

Pour aider à répondre à cette question, il faut utiliser l'outil statistique. Il est préférable de prévoir une collaboration avec des unités spécialisées en épidémiologie professionnelle, même lors d'études réalisées exclusivement en milieu de travail. Les cadres de partenariat ou de formation sont aussi des bonnes réponses à la nécessaire maîtrise des outils statistiques.

\section{Cadres réglementaires.}

Les évaluations pratiquées dans le cadre d'enquêtes épidémiologiques relèvent en général de dispositions réglementaires. D'une part, la réalisation d'une enquête épidémiologique implique quasiment toujours la constitution de fichiers de données directement ou indirectement nominatives : la mise en place de ces fichiers nécessite l'accord du Comité consultatif sur le traitement de l'information en matière de recherche dans le domaine de la santé d'une part, et de la Commission nationale de l'informatique et des libertés (CNIL) d'autre part.

De plus, en cas d'explorations médicales réalisées spécifiquement pour une étude, l'enquête est soumise à la loi relative à la protection des personnes qui se prêtent à la recherche biomédicale (loi Huriet). Une étape supplémentaire oblige alors l'investigateur à soumettre son projet au Comité consultatif pour la protection des personnes qui se prêtent à la recherche biomédicale (CCPPRB), avant toute autre démarche. L'investigateur doit recueillir un consentement éclairé du salarié par écrit, après lui avoir transmit une information claire, loyale au salarié sur les objectifs et les moyens de l'enquête, sur la totale liberté sur l'adhésion du salarié volontaire à ladite enquête et sur la possibilité de se rétracter à tout moment. Celui-ci doit signer une notice précisant qu'il a reçu et compris l'information sur l'étude. L'ensemble des autorisations doit être obtenu avant que le travail de terrain ne commence. Toutes ces instances réglementaires se prononcent sur la base des éléments consignés dans le protocole.

\section{Quel type d'enquête?}

Il existe plusieurs types d'enquêtes épidémiologiques, dont les enquêtes descriptives et les enquêtes analytiques, le choix de l'étude étant fonction des objectifs et des moyens disponibles (6). Les études expérimentales (dont les études d'intervention) et les systèmes de surveillance ne seront pas abordés ici. Les études descriptives, analytiques transversales et de cohorte historique sont les plus fréquemment réalisées en entreprise $(7,8)$. 


\section{Enquêtes descriptives}

Les études épidémiologiques descriptives étudient la fréquence et la répartition de paramètres de santé et/ou de facteurs de risque dans les populations. Elles comportent des mesures de l'état de santé (mortalité, morbidité) et une évaluation de l'exposition et des conditions de travail. Ces études peuvent concerner un phénomène à un moment donné (de manière transversale) ou porter sur plusieurs points (cohorte ou étude transversales répétées), ce qui permet d'étudier des variations temporelles.

Les données recueillies peuvent être mises en perspective avec celles d'une population de référence, pour soulever l'existence de possibles associations. Les comparaisons prennent alors en compte des valeurs de références disponibles à niveau plus général. Un exemple est celui des comparaisons à des données de références nationales ou régionales, rendues disponibles par différents organismes comme l'INSERM, l'Institut national des statistiques et des études économiques (INSEE), l'Institut national pour la recherche et la sécurité (INRS), le National institut of occupational and safety of health (NIOSH) par exemple.

Ces études sont les plus simples et les plus faciles à organiser. Elles nécessitent cependant la même rigueur méthodologique que les autres types d'étude. En règle générale, l'interprétation de ce type d'enquête doit être excessivement prudente : il ne sera pas possible de conclure à l'existence d'un facteur favorisant et, a fortiori, causal de pathologies ; ces études sont néanmoins utiles pour la mise en évidence d'un problème particulier en entreprise et sont souvent utilisées comme point de départ permettant de formuler des hypothèses pour la mise en place d'études analytiques spécifiques ultérieures. Par exemple, suite à un accident de travail, une enquête descriptive sur les conditions de travail des salariés peut mettre en évidence un excès de certaines pathologies et décrire parallèlement la répartition de certains facteurs susceptibles de leur être associées. Une enquête analytique est ensuite nécessaire pour confirmer ou infirmer un éventuel lien évocateur de causalité entre ces faits.

La principale source d'erreur de ces études réside dans le choix de la population étudiée, notamment en cas de comparaison avec une population type.

\section{Enquêtes analytiques}

La plupart des enquêtes analytiques reprennent souvent, en première partie des résultats, la description de la population d'étude, de la distribution de la fréquence ou de l'intensité de l'exposition et des pathologies étudiées.

Les enquêtes analytiques, appelées aussi étiologiques, consistent à comparer des groupes de sujets pour mettre en évidence une association entre une exposition et une pathologie. Cette association est quantifiée par le risque relatif ou l'odds ratio selon le type d'étude. Ce chiffre s'il est significativement 
supérieur à 1 indique que l'exposition à ce facteur est statistiquement associée positivement à la maladie étudiée (a contrario un odds ratio strictement inférieur à 1 évoque un facteur associé à la sous-incidence, dit protecteur). On distingue trois types d'enquêtes analytiques : les enquêtes transversales, les enquêtes cas-témoins et les enquêtes de cohortes. Ce qui différencie ces trois types d'enquêtes c'est le moment d'inclusion et le type d'information recueillie ; les enquêtes transversales concernent la situation à un instant donné ; le recueil de l'information concerne l'exposition et la maladie au moment de l'inclusion des sujets. Dans les enquêtes cas-témoins, l'inclusion des sujets se fait après le diagnostic de la maladie et le recueil d'information est rétrospectif, c'est-à-dire qu'il documente une exposition antérieure à l'inclusion des sujets. Ces dernières sont néanmoins plus rares en milieu de travail. Dans une étude de cohorte, l'information sur l'exposition est contemporaine de l'inclusion de sujets indemnes de la pathologie étudiée et les informations sur l'évaluation de l'exposition et la santé des sujets sont recueillies ensuite au fil du suivi.

\section{- Enquêtes transversales.}

Elles sont caractérisées par un échantillon issu, soit de l'ensemble de la population, soit d'un échantillon, sans sélection ni sur l'exposition ni sur la maladie. Ainsi les sujets inclus dans l'étude sont tous ceux qui sont présents au moment de l'enquête. Parmi les enquêtes transversales, certaines sont des enquêtes transversales descriptives, d'autres sont de type étiologique.

Ces enquêtes sont caractérisées par leur facilité d'organisation et leur faible coût. Elles posent le problème de la difficulté d'établir la relation temporelle entre le facteur de risque et la pathologie, ainsi que celui des sujets absents lors du recrutement. Le choix de l'échantillon peut ainsi conduire à des erreurs ou biais sur la représentativité de l'échantillon (9). Les résultats sont donc difficiles à interpréter en termes de causalité.

\section{- Enquêtes cas-témoins.}

Le principe des enquêtes cas-témoins est de comparer l'exposition antérieure chez des sujets malades (les cas) à l'exposition de témoins, sujets représentatifs de la population d'où sont issus les cas et indemnes de la pathologie étudiée. Dans ces enquêtes, le recueil d'information concerne essentiellement l'exposition, qui est par définition rétrospectif.

Ces enquêtes présentent l'avantage de pouvoir inclure un nombre plus faible de sujets, ce qui est particulièrement intéressant pour étudier une pathologie rare. D'autre part elles ne nécessitent pas de suivi dans le temps. Parfois plus coûteuses que les enquêtes transversales, elles restent généralement moins onéreuses que les enquêtes de cohorte. Elles permettent par ailleurs d'étudier simultanément 
plusieurs facteurs de risque d'une maladie.

Les difficultés principales des enquêtes cas-témoins reposent sur le choix de la population témoin qui doit être aussi comparable que possible à la population des cas en ce qui concerne les facteurs que l'on ne désire pas étudier, notamment les facteurs connus pour être liés à la maladie (âge, sexe en particulier). Il existe également la difficulté de reconstituer de façon fiable et précise une exposition antérieure et parfois ancienne (biais de mémoire). Par ailleurs, ce type d'enquête ne permet pas d'estimer l'incidence de la maladie. L'intensité des associations entre la maladie et les expositions est estimée par des odds ratios (notés OR).

\section{- Enquêtes de cohorte.}

Une cohorte est définie comme un groupe de sujets suivis au cours du temps. Ce type d'enquête prend souvent le nom d'enquête longitudinale. Un exemple bien connu est celui des enquêtes exposés/non-exposés. Le but des études de cohorte est de comparer la morbidité ou la mortalité de sujets exposés à un facteur particulier à celle que l'on observe chez des sujets non exposés à ce facteur. Les avantages de ce type d'étude sont une meilleure documentation de l'exposition, la possibilité d'étudier plusieurs pathologies. Elles permettent en outre une estimation directe des taux d'incidence et donc du risque relatif de maladie.

Les inconvénients principaux sont leur coût élevé lié aux effectifs et à la durée du suivi, d'autant plus importants que l'incidence de la pathologie est faible, et les inévitables perdus de vue, c'est-à-dire les personnes dont on cesse d'avoir des nouvelles avant la fin de l'étude.

Les études de cohorte peuvent être prospectives; le début de l'étude coïncide alors avec le moment de l'exposition des sujets. Elles peuvent être rétrospectives, également, et sont alors appelées cohortes historiques: il s'agit de reconstituer, à partir de données archivées, l'histoire professionnelle et médicale des sujets. Lorsqu'elles sont réalisables, elles présentent de nombreux avantages notamment pour l'étude des risques professionnels: obtention plus rapide de résultats pour des pathologies ayant un temps de latence important et surtout pour des expositions rares en dehors du milieu professionnel. Bien entendu, ce type d'étude comporte un risque de biais (voir plus loin le paragraphe consacré à ce thème) lié à la sélection des sujets, selon que leur histoire professionnelle et médicale présente des données manquantes.

\section{- Enquêtes mixtes.}

Il existe également des enquêtes dites mixtes ou hybrides, telle que les enquêtes cas-témoins nichées au sein d'une cohorte (où cas et témoins sont issus des sujets de la cohorte), les enquêtes transversales répétées dans le temps, ou encore les études de cohorte dont le point de départ est une étude transversale. 
Notre étude a repris les données d'une enquête analytique comportant une partie transversale en 1993-94 et une autre longitudinale avec un suivi de 1993-94 à 1996-97. Les résultats de cette vaste enquête ont permis de mettre en évidence des facteurs de risque, outre du syndrome du nerf ulnaire au coude qui nous sert d'exemple, d'épaule douloureuse, d'épicondylites et de syndrome du canal carpien $(3,5,10-15)$.

\section{Population d'étude}

La population cible est celle dont on veut pouvoir extrapoler les résultats. La population source est celle dont l'échantillon est extrait lors de la réalisation pratique de l'étude. La définition de la population cible peut être plus complexe qu'il n'y paraît: on peut par exemple chercher à mettre en évidence un résultat général (c'est-à-dire extrapolable à toute une population) à partir d'une population d'étude particulière. Les résultats d'une étude passent d'abord par l'extrapolation de l'échantillon à la population source, puis de la population source à la population cible, si ces deux dernières diffèrent (très souvent ces populations sont très proches voire identiques).

La plupart des études épidémiologiques ne portent pas sur l'ensemble de la population considérée, à l'exception de certaines enquêtes descriptives dans des petites entreprises. Il faut constituer un échantillon adapté à la question posée, l'échantillon le meilleur n'étant pas toujours un échantillon représentatif ; la question du choix de l'échantillon justifie le plus souvent l'avis d'un méthodologiste statisticien ou épidémiologiste.

Les critères de choix de l'échantillon sont différents en fonction du type d'étude.

La taille de l'échantillon peut être calculée à partir des connaissances existantes sur le sujet à partir de la bibliographie, ainsi que de la puissance désirée de l'enquête (c'est-à-dire la probabilité de mettre en évidence une différence définie minimale si elle existe).

La population cible de l'étude TMS était la population exposée aux gestes répétitifs. La population source était celle de salariés surveillés par 18 médecins du travail répartis dans six régions de France.

L'échantillon de la cohorte était constitué par 700 salariés. Or, 102 de ces salariés n'ont pu être étudiés trois ans après car perdus de vue. Considérant que l'étude s'attache à déterminer les facteurs liés à l'incidence, les 20 salariés déjà atteints d'un syndrome du nerf ulnaire au coude en 1993-94 n'ont pas été inclus dans l'étude. L'effectif de la cohorte était donc de 578 salariés. Cet exemple met en évidence le décalage entre les effectifs prévus dans le protocole et ceux effectivement disponibles au moment de l'analyse. De plus, ici, les effectifs avaient été calculés pour étudier les pathologies les plus fréquentes (épaule douloureuse et canal carpien notamment), et auraient pu se révéler insuffisants pour étudier une pathologie plus rare comme le syndrome du nerf ulnaire au coude. 


\section{Recueil de données et définition des critères de jugement}

La définition de la maladie, de l'exposition et des autres variables doit être précise, mais surtout standardisée, pour permettre une bonne comparabilité entre les groupes. L'utilisation d'outils standardisés déjà éprouvés lors d'autres enquêtes nationales et internationales permet une comparabilité avec d'autres études.

\section{Définition de la maladie}

Avant de débuter une étude, il est nécessaire de préciser comment la pathologie ou l'état de santé que l'on va étudier seront évalués. Le milieu du travail français bénéficie d'un investigateur formé aux risques professionnels : le médecin du travail qui est de ce fait un investigateur privilégié de ce type d'étude.

La (ou les) pathologie(s) concernée(s) par l'enquête doit(vent) être définie(s) de manière précise avec des critères stricts. La définition des cas doit s'appuyer sur des critères reproductibles, sensibles et spécifiques. En règle générale, il est préférable d'utiliser les critères consensuels issus de la bibliographie. Suivant la pathologie étudiée, ces critères peuvent s'appuyer sur des autoquestionnaires, des interrogatoires (échelles utilisées en psychiatrie), des critères cliniques standardisés, voire des mesures ou des prélèvements, ou d'autres explorations (électromyogramme, dosages, histologie ...). Dans le cas particulier des enquêtes de cohorte, il est indispensable de définir préalablement les modalités pratiques des examens réalisés lors du suivi (systématique/volontariat, délai, convocations et rappels...).

Dan l'étude TMS, la pathologie étudiée était le syndrome du nerf ulnaire au coude. Les médecins $d u$ travail ont pratiqué un examen standardisé pour chaque salarié, basé sur des critères diagnostiques cliniques consensuels, assurant une bonne comparabilité.

\section{Définition de l'exposition}

De nombreux moyens d'évaluation de l'exposition sont disponibles en milieu de travail : la fiche de poste (complétée par des informations toxicologiques, par exemple des fiches de données de sécurité), ainsi que le calendrier professionnel de chaque salarié généralement conservé par le médecin du travail ; d'autre part, des données d'exposition indirecte (métrologie) et directe (biométrologie) peuvent être disponibles, soit de manière systématique, soit recueillies dans le cadre de l'étude. Des 
questionnaires, des examens complémentaires, des expertises, des matrices emplois-exposition (matrices réalisées, soit en entreprise, soit en population générale, qui permettent d'attribuer à une profession une probabilité d'exposition à certaines substances définies) peuvent être utilisés en fonction de l'étendue de l'enquête (2). En règle générale, il est préférable de quantifier l'exposition de la manière la plus détaillée possible au moment du recueil, afin d'étudier de possibles relations doseeffets. Si la précision sur la qualification de l'exposition est un aspect important, d'autres qualités des mesures sont également requises dont notamment leur comparabilité (même niveau de précision sur l'exposition pour tous les sujets étudiés). Les questions de faisabilité (coût et acceptabilité par les salariés des explorations pratiquées ...) sont aussi à prendre en compte.

Les problèmes liés à la reconstitution d'exposition avant le début de la maladie sont spécifiques aux enquêtes rétrospectives, études cas témoins et cohortes historiques essentiellement. L'idéal serait de disposer de l'ensemble des informations concernant l'exposition dans le dossier médical. Néanmoins, il est le plus souvent nécessaire de compléter l'évaluation a posteriori, grâce à l'emploi de questionnaires, ou d'évaluer l'exposition grâce à la connaissance du parcours professionnel du sujet et d'une matrice emplois-expositions lorsqu'elle existe pour le produit et le secteur d'activité considéré. Les questionnaires devront être standardisés de manière à être identiques pour tous et administrés, autant que possible, indépendamment de l'état de santé du sujet (enquêteur indépendant et aveugle de l'état de santé du sujet). L'objectif est d'éviter au maximum les erreurs de classement des sujets, notamment les erreurs qui affectent différemment les sujets malades de sujets indemnes.

Dan l'étude TMS, l'exposition aux gestes répétitifs a été définie par le médecin du travail lui-même, et vérifiée par auto questionnaire rempli par le salarié sur ses conditions de travail. Une quantification de la répétitivité (évaluée par le temps de cycle) faisait aussi partie des données recueillies.

\section{Notion de biais.}

Avant le début de l'enquête, il est indispensable de prévoir et de décrire l'ensemble des étapes précédemment évoquées. Ainsi, une méthodologie rigoureuse permet de limiter ou du moins d'anticiper la survenue de biais. Le biais est un effet qui tend à produire une estimation systématiquement différente, en plus ou en moins, de la vraie valeur du paramètre étudié. Les principaux biais des études épidémiologiques sont énumérés dans le tableau I. Ils peuvent survenir lors de la sélection de l'échantillon (biais de sélection), lors du classement des cas et des sujets sains selon l'exposition ou lors de la prise en compte de l'exposition (biais de classement). Par ailleurs, un facteur, que l'on ne désire pas étudier en lui-même, mais lié à la fois à la maladie et à l'exposition, peut induire, s'il n'est pas pris en compte, une erreur d'interprétation (biais de confusion). C'est pour cela que d'autres informations, dépendant des connaissances sur le sujet étudié, doivent être collectées. 


\section{Autres informations}

En dehors de données sur l'exposition et sur la pathologie, il est aussi indispensable de collecter dans le même temps des informations d'ordre sociodémographique et sur d'éventuels facteurs dits de confusion (c'est-à-dire associés à la fois à l'exposition et à la maladie étudiée). Les facteurs associés éventuels sont répertoriés lors de l'étude bibliographique. Il existe plusieurs manières de les prendre en compte (en dehors du tirage au sort qui sort du cadre de ce travail), parfois dès la planification de l'étude.

- Sélection d'une population à risque : il est possible de restreindre la population de l'étude, en sélectionnant une population où des facteurs de confusion ne sont pas présents (rarement possible en pratique).

- Appariement : il est possible d'apparier les deux groupes que l'on veut comparer sur des facteurs de confusion potentiels.

Au moment de l'analyse, des techniques statistiques d'ajustements et de stratifications permettent également de tenir compte de certains facteurs associés.

Dans notre étude, plusieurs points ont dî être vérifiés pour éviter l'introduction de biais.

Comme dans la plupart des études en milieu professionnel, la population étudiée est une population de personnes au travail, donc sélectionnés pour être indemnes de maladie grave et incapacitante. Il s'agit de l'effet du travailleur sain ("Healthy Worker Effect"). Néanmoins, comme l'objectif est de déterminer les facteurs de risque de syndrome du nerf ulnaire chez les travailleurs, il n'y a pas de biais de sélection. On peut cependant se demander si les résultats sont extrapolables à l'ensemble des salariés de ce type d'activité. Les caractéristiques des sujets qui n'ont pas été réexaminés en 1996-97 et notamment les raisons de non-réponse, ont aussi été vérifiées pour contrôler un éventuel biais de sélection lié aux perdus de vue.

La standardisation de l'exposition et de l'examen clinique (par des questionnaires et tests cliniques précis et identiques quel que soit le poste de travail) ont permis de minimiser des erreurs liées au classement différent des sujets selon les investigateurs (18 médecins du travail différents).

Enfin prenons l'exemple de l'âge qui est un facteur de confusion éventuel. Les affections d'hypersollicitation du membre supérieur sont plus fréquentes chez les salariés âgés et il est concevable que les salariés plus âgés déclarent plus souvent travailler en force. Dans notre analyse, il a donc été nécessaire de prendre en compte ce facteur associé, le principe étant d'étudier les relations entre les affections d'hypersollicitation du membre supérieur et le travail en force à âge constant. 


\section{Mise en place du recueil de données et aspects logistiques}

Les modalités pratiques du recueil de données doivent être définies (investigateur, date, support...). Un suivi régulier de l'avancement du recueil de données est généralement organisé sous forme de cahier de suivi ou équivalent informatisé, avec notamment, mention des dates des examens et de questionnaires et des dates des relances. En cas d'enquête prospective prolongée, il est recommandé d'organiser des réunions périodiques avec les participants de l'étude afin d'éviter un désistement progressif.

Des partenaires incontournables dans les entreprises qui en sont pourvues sont les infirmières du travail et les secrétaires médicales. Elles représentent souvent une aide précieuse pour le médecin investigateur sur le plan logistique.

Les questionnaires seront anonymisés et conservés précieusement.

La saisie de donnée peut prendre plusieurs formes suivant les moyens de l'étude. En cas d'enquêtes multicentriques nationales, la saisie de données est souvent réalisée moyennant contrat par des organismes spécialisés. En cas d'effectifs réduits et d'enquêtes réalisées avec un petit budget, le médecin du travail peut saisir lui-même les données (ou grâce à une personne de son service), soit sur des logiciels spécialisés (Epi-Info* ou SPSS*), soit des logiciels multi usages (Excel*, en exportant ensuite les données dans un logiciel statistique). On préféra des logiciels spécialisés qui permettent d'effectuer des contrôles au moment de la saisie.

Les données TMS ont été centralisées d'abord par l'inspection médicale du travail puis par l'unité INSERM et saisies par un organisme indépendant. Un numéro a été affecté à chaque sujet au début de l'enquête. C'est ce numéro qui apparaît sur les questionnaires, par conséquent rendus anonymes. Les documents papiers et informatiques anonymisés sont conservés par l'unité INSERM.

\section{Analyse et interprétation}

\section{Quels résultats?}

Les premiers résultats comportent avant tout la description de l'échantillon et les écarts éventuels au protocole ou à l'enquête idéale : taux de refus, perdus de vue, données incomplètes ... Quand les résultats portent sur des données déjà largement analysées, la partie «description des données » est réduite ; les auteurs font alors référence aux articles antérieurs portant sur les mêmes données.

Ensuite, se décline la présentation des résultats de l'analyse et les diverses comparaisons, dont notamment la réponse à la question posée (ou aux questions posées) constituant l'objectif de l'enquête. 
Dans notre exemple sur le syndrome du nerf ulnaire au coude, grâce à des tests de khi2, plusieurs variables statistiquement liées à cette pathologie ont été sélectionnées. Après analyse statistique, parmi les variables professionnelles sélectionnées, seule l'« utilisation d'un outil en force " s'est avérée significativement associée à l'incidence du syndrome du nerf ulnaire au coude avec un odds ratio à 4,1 et un intervalle de confiance à 95\% [1,4-12,0] ce qui permet de conclure que cette exposition professionnelle est associée à une augmentation du risque de survenue de la maladie.

\section{Quelles conclusions?}

Il est possible de conclure sur la question posée par l'interprétation des résultats ; par exemple, les analyses statistiques ont permis de mettre en évidence une relation entre une exposition et une maladie. Néanmoins la généralisation de ces résultats doit prendre en considération l'existence d'éventuels biais. De plus, une association entre une exposition et une maladie n'implique pas de lien de causalité direct. L'interprétation causale doit intégrer la validité de l'étude, mais aussi sa cohérence interne (forte association, spécificité, relation dose-effet et temps-effet) ainsi que sa cohérence externe (consistance, reproductibilité, plausibilité ...).

La partie discussion des articles scientifiques permet de comparer les résultats avec les données de la littérature, d'expliciter clairement la validité des résultats et de discuter les sources de biais potentiels. En règle générale, les revues scientifiques veillent à ce que les articles publiés soient solides sur le plan méthodologique. Pour ce faire, elles font relire le manuscrit (en général) par deux relecteurs (dits reviewers en anglais) qui peuvent suggérer des modifications, ou donner un avis négatif sur la publication. En épidémiologie, les résultats d'une étude sont à considérer dans un contexte plus général, faisant référence à d'autres études épidémiologiques et à des connaissances éventuellement apportées par d'autres disciplines.

\section{Conclusion}

Le but de cet article n'était pas d'être exhaustif sur l'épidémiologie des risques professionnels dont il existe des spécialistes en France, mais d'envisager trois points essentiels pour le médecin du travail:

- faciliter la lecture critique d'articles épidémiologiques sur les risques professionnels ;

- donner les bases et les motivations pour participer à des enquêtes épidémiologiques de portée nationale ;

- enfin, envisager la possibilité d'organiser des enquêtes spécifiques au sein des entreprises qu'il surveille. 


\section{Références}

[1] Goldberg M., et al. L'épidémiologie sans peine. 2 ed. Paris: Frison-Roche, 1990.

[2] Bouyer J., Hémon D., Cordier S., al. Epidémiologie.Principes et méthodes quantitatives. Paris : INSERM, 1995.

[3] Descatha A, Leclerc A, Chastang JF, Roquelaure Y. Incidence of ulnar nerve entrapment at the elbow in repetitive work. Scand J Work Environ Health 2004; 30:234-240.

[4] Goldberg M., et coll. Guide des recommandations de bonnes pratiques en épidémiologie. ADELF, ADEREST. 2ed 2004.

[5] ANACT, Inserm U88, INRS. Affections périarticulaires des membres supérieurs et organisation du travail. Résultats de l'enquête épidémiologique nationale. Documents pour le médecin du travail 1996; 65:13-31.

[6] Leclerc A., Lellouch J. Glossaire statistique et épidemiologique. Encycl Med Chir (Elservier Paris), Toxicologie-Pathologie professionnelle, 15. 2000.

[7] Bardot F., Bardouillet M.C., Boitel L., Buisset C., Castel M.J., Jabo F. et al. SVP50: une enquête sur les enjeux de santé au travail pour les salariés de plus de 50 ans. Arch Mal Prof 2004; 65:232-233.

[8] Bardouillet M.C., Gey C., Hamonic T., Jorry F. Devenir médico-professionnel des opérés du canal carpien. Arch Mal Prof 2003; 64:83-88.

[9] Rumeau-Rouquette C., Blondel B., Kaminski M., Bréart G. Epidemiologie. Méthodes et pratique. 5 ed. Paris: Flammarion médecine-sciences, 1999.

[10] Leclerc A, Franchi P, Cristofari MF, Delemotte B, Mereau P, Teyssier-Cotte C et al. Carpal tunnel syndrome and work organisation in repetitive work: a cross sectional study in France. Study Group on Repetitive Work. Occup Environ Med 1998; 55:180187.

[11] Leclerc A, Landre MF, Chastang JF, Niedhammer I, Roquelaure Y. Upper-limb disorders in repetitive work. Scand J Work Environ Health 2001; 27:268-278.

[12] Leclerc A, Chastang JF, Niedhammer I, Landre MF, Roquelaure Y. Incidence of shoulder pain in repetitive work. Occup Environ Med 2004; 61:39-44.

[13] Descatha A, Leclerc A, Chastang JF, Roquelaure Y. Medial epicondylitis in occupational settings: prevalence, incidence and associated risk factors. J Occup Environ Med 2003; 45:993-1001.

[14] Descatha A, Leclerc A, Chastang JF, Roquelaure Y. Epicondylites médiales : évolution et prévention. Arch Mal Prof 2003; 64:235-245. 
[15] Niedhammer I, Landre MF, Leclerc A, Bourgeois F, Franchi P, Chastang JF et al. Shoulder disorders related to work organization and other occupational factors among supermarket cashiers. Int J Occup Environ Health 1998; 4:168-178.

Quelques sites :

Pubmed : http://www.ncbi.nlm.nih.gov/entrez/query.fcgi

NIOSH: http://www.cdc.gov/niosh/homepage.html

ADELF: http://adelf.isped.u-bordeaux2.fr/

INRS : http://www.inrs.fr/

InVS : http://www.invs.sante.fr/

INSERM : http://www.inserm.fr/

CNIL : http://www.cnil.fr/

INSEE : http://www.insee.fr/

Banque de donnée de santé publique: http://www.bdsp.tm.fr/

Tableau I : Biais et sources de biais.

\begin{tabular}{|c|c|}
\hline Type de biais & Exemples de source de biais \\
\hline Sélection de l'échantillon & $\begin{array}{l}\text {-Participation biaisée (auto sélection) } \\
\text {-Volontaires non-répondeurs } \\
\text {-Effet du travailleur sain } \\
\text {-Perdus de vue }\end{array}$ \\
\hline Erreurs de classement (=d'information) & 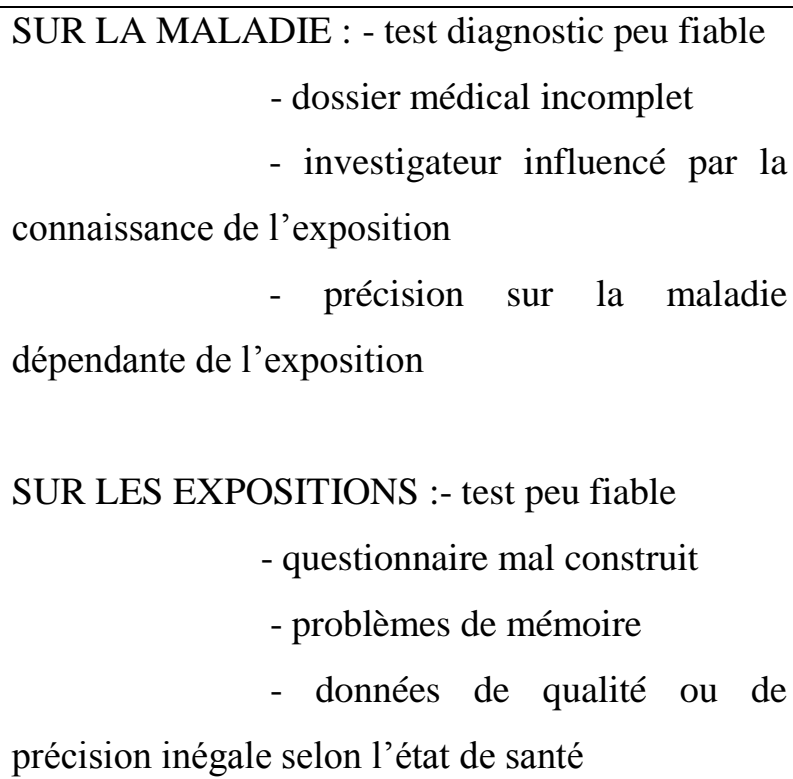 \\
\hline Facteurs de confusion & $\begin{array}{l}\text { Facteur lié à la fois à la maladie et au facteur de } \\
\text { risque. }\end{array}$ \\
\hline
\end{tabular}

